\title{
Pros and cons of symmetrical dual- $k$ spacer technology in hybrid FinFETs
}

\author{
K.P. Pradhan ${ }^{a}{ }^{*}$, M.G.C. Andrade ${ }^{\text {b }}$, P.K. Sahu ${ }^{\text {a }}$ \\ a Nano-Electronics Laboratory, Department of Electrical Engineering, National Institute of Technology, Rourkela, Odisha, 769008, India \\ b UNESP - Univ Estadual Paulista, Group of Automation and Integrated Systems, Av. Trs de Maro, n. 511, 18087-180, Sorocaba, Brazil
}

\section{A R T I C L E I N F O}

\section{Article history:}

Received 22 September 2016

Accepted 27 September 2016

Available online 29 September 2016

\section{Keywords:}

Trigate FinFET

Hybrid FinFET

Symmetrical dual- $k$ spacer

Analog/RF

Short channel effects (SCEs)

\begin{abstract}
A B S T R A C T
The symmetrical dual- $k$ spacer technology in hybrid FinFETs has been widely explored for better electrostatic control of the fin-based devices in nanoscale region. Since, high- $k$ tangible spacer materials are broadly became a matter of study due to their better immunity to the short channel effects (SCEs) in nano devices. However, the only cause that restricts the circuit designers from using high- $k$ spacer is the unreasonable increasing fringing capacitances. This work quantitatively analyzed the benefits and drawbacks of considering two different dielectric spacer materials symmetrically in either sides of the channel for the hybrid device. From the demonstrated results, the inclusion of high- $k$ spacer predicts an effective reduction in off-state leakage along with an improvement in drive current. However, these devices have paid the cost in terms of a high total gate-togate capacitance $\left(C_{g g}\right)$ that consequently results poor cutoff frequency $\left(f_{T}\right)$ and delay.
\end{abstract}

(c) 2016 Elsevier Ltd. All rights reserved.

\section{Introduction}

The invention of 3-D FinFET technology in the semiconductor era has been considerably enabled the advancement of electronics industry and have been of immense interest for sub-20 $\mathrm{nm}$ applications [1,2]. The fin based devices are excellent in suppressing the short channel effects (SCEs) and have superior control over the carriers in the channel [3-7]. This improvement in technology facilitates a longer battery lifetime and energy efficient electronics in both operating regions i.e., high performance (HP) and low power (LP). According to the reported articles and International Technology Roadmap for Semiconductors (ITRS) prediction, the 3-Dimensional topology is here to expedite the profitable industries likely to 5-nm technology node $[8,9]$. The only modifications in lower nodes is either in physical architecture like nanowires and nanotubes or incorporation of new materials in the channel such as Ge, GaAs, SiGe, etc. keeping the CMOS compatibility [10-12]. Today's market is more consensus about the HP consumer applications and may continue for subsequent years. The HP applications primary goal is to achieve high current drivability with higher packing density instead of bothering regarding the static leakage current $[11,13]$. So, to achieve the HP computing applications, a new transistor i.e., hybrid FinFET was first proposed by Zhang et al. [14]. Henceforth further modification and continuous research on hybrid devices was carried by Fahad et al. [15,9] and Pradhan et al. [11,16,17]. Hybrid FinFETs merge several technologies in a single SOI platform namely 3-D FinFET and 2-D UTB MOSFET. However, to analyze further impact of spacer technology in the hybrid FinFETs, this work incorporates two different spacer materials symmetrically in the underlap region of either side of the channel for the hybrid

\footnotetext{
* Corresponding author.

E-mail addresses: k.p.pradhan@ieee.org (K.P. Pradhan), gloria@sorocaba.unesp.br (M.G.C. Andrade), pksahu@nitrkl.ac.in (P.K. Sahu).
} 
device. Previously, the underlap FinFETs have showed immense control over SCEs [18,19]. But the major issues, which are faced by underlap FinFETs are the precise control of doping profile and an increased amount of underlap resistance. These issues are well tackled by Refs. [18-20] with an inclusion of high- $k$ dielectric spacers at the underlap region. Similarly, Pal et al. $[21,7]$ have examined that superior control on the channel and meaningful improvements are achievable in terms of $I_{o n}$ and $I_{o f f}$ by contemplating dual-k spacers over the underlap regions. The above mentioned works are carried out contemplating the high- $k$ spacer materials in the conventional 3-D FinFET structures. In this work, we have estimated the benefits as well as drawbacks of symmetrical inclusion of dual- $k$ spacer in either side of the channel for the hybrid devices over conventional FinFETs. The lengths of both spacers (inner high- $k, L_{h k}$, and outer low- $k, L_{l k}$ ) are optimized for better circuit applications. The performances considered for this investigation are drive current $\left(I_{o n}\right)$, off state leakage current $\left(I_{o f f}\right)$, effective drain current $\left(I_{D E F F}\right)$, subthreshold slope $(S S)$, transconductance $\left(g_{m}\right)$, total gate capacitance $\left(C_{g g}\right)$, output conductance $\left(g_{d}\right)$, cutoff frequency $\left(f_{T}\right)$, and gain $\left(A_{V}\right)$.

The organization of the paper is as follows: Section 2 describes the architecture of hybrid FinFETs. Section 3 presents the simulation setup that discusses the methods and models considered for simulating the devices. In Section 4, the investigation is done for optimizing the length of dual- $k$ spacer and the benefits and drawbacks of the high- $k$ spacer inclusion. Finally, the concluding observations are pointed in Section 5.

\section{Hybrid FinFET architecture with symmetrical dual-k spacer technology}

The 3-D bird view of conventional SOI FinFET and hybrid FinFET with dual- $k$ spacer technology architectures chosen for this work are presented in Fig. 1 (a) and (b) accordingly. Fig. 1(c) demonstrates the vertical and horizontal 2-D perspective views of both the devices. The SOI FinFET [6] offers significant control over SCEs and electrostatic parameters with a vertical thin channel and also have lesser fabrication complexity as compared to the bulk FinFET [22]. The major issues in SOI FinFET are self-heating effects because of the thick buried oxide and the cost factor. However, the self-heating effect is well-taken care by Fiegna et al. [23] and the cost factor is mitigated by the less complicated process steps. The 3-D FinFET and 2-D UTB technology are combined together to design the advanced hybrid topology. It predicts many advantages like maximum area efficiency, high packing density, and high drive current as studied in Refs. [14,9]. The key objective in this work is to establish symmetrical underlap regions at both sides of the channel towards source/drain and pattern the regions with two different dielectric spacer materials $\left(\mathrm{Si}_{3} \mathrm{~N}_{4}, k=7.5\right.$ and $\left.\mathrm{HfO}_{2}, k=22\right)$. Then the benefits and drawbacks in performances of the designed device are compared with conventional FinFET [6]. The spacer lengths of $\mathrm{Si}_{3} \mathrm{~N}_{4}$ is termed as $\mathrm{L}_{l k}$ and $\mathrm{HfO}_{2}$ as $\mathrm{L}_{h k}$ and the ratio of $L_{l k}: L_{h k}$ is varied to get an optimum ratio. The physical and electrical parameters are calibrated according to the ITRS specifications $[8,6]$. The $\mathrm{SiO}_{2}$ with physical thickness of $0.9 \mathrm{~nm}$ is considered as gate oxide.

The channel is lightly doped to prevent the device from random dopant fluctuation (RDF) effect. Table 1 shows the physical dimensions for designing both the devices. Several technologies are merged in this work i.e., 3-D FinFET + 2-D UTB + spacer

(a)



(b)
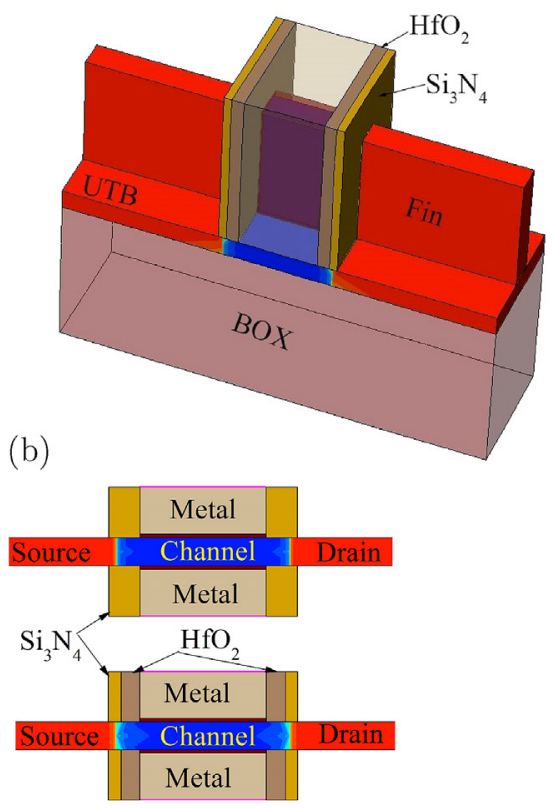

Fig. 1. (a) Typical trigate FinFET (D1) (b) Symmetrical dual-k spacer hybrid FinFET (D2, D3, D4, D5) (C) 2-D cut view of both devices. 
Table 1

${ }^{a}$ Device design parameters.

\begin{tabular}{llll}
\hline Parameters & Nomenclature $(\mathrm{nm})$ & Conventional FinFET & Symmetrical Dual- $k$ hybrid FinFET \\
\hline$W_{\text {Fin }}$ & Fin Width & $7 \mathrm{~nm}$ & $7 \mathrm{~nm}$ \\
$H_{\text {Fin }}$ & Fin Height & $30 \mathrm{~nm}$ & $H_{\text {Fin }}-\mathrm{UTB}=25 \mathrm{~nm}$ \\
$L_{g}$ & Gate length & $20 \mathrm{~nm}$ & $20 \mathrm{~nm}$ \\
UTB & Ultra thin body thickness & - & $5 \mathrm{~nm}$ \\
$L_{l k}: L_{h k}$ & Length of low- $k:$ Length of high- $k$ & $5: 0(\mathrm{D} 1)$ & $1: 4(\mathrm{D} 2), 2: 3(\mathrm{D} 3), 3: 2(\mathrm{D} 4), 4: 1(\mathrm{D} 5)$ \\
$t_{\text {ox }}$ & physical gate oxide thickness & $0.9 \mathrm{~nm}$ & $0.9 \mathrm{~nm}$ \\
$L_{T}$ & Total device length & $110 \mathrm{~nm}$ & $110 \mathrm{~nm}$ \\
$W_{T}$ & Total device width & $32.2 \mathrm{~nm}$ & $32.2 \mathrm{~nm}$ \\
BOX & Buried oxide thickness & $40 \mathrm{~nm}$ & $40 \mathrm{~nm}$ \\
\hline
\end{tabular}

a 2013 Overall roadmap technology characteristics (ORTC) ITRS parameters for $14 \mathrm{~nm}$ technology node FinFET.

engineering (two different dielectrics as inner high- $k$ near to channel and outer low- $k$ near to S/D in the underlap region). And a list of benefits and drawbacks for the adopted technology over conventional FinFET are systematically presented.

\section{Simulation techniques}

Comprehensive 3-D simulations are being carried out by Sentaurus TCAD [24] to evaluate the prospective benefits and drawbacks of the hybrid device over traditional one. The numerical process and device simulations allow crucial insights on the nature of semiconductor devices that can lead to new perceptions [24]. However, the simulation needs to be properly calibrated before designing any device. Hence, the validity of the simulator has been established according to our previous work [16]. The mobility model that accounts of doping, transverse field, and velocity saturation dependency along with the drift-diffusion (DD) model are considered in the simulation. The quantum confinement effect in fin and UTB which is determined from the density gradient based quantization model is also activated in the simulation. The Lombardi high- $k$ model is considered to dealt with the high- $k$ related mobility degradation effects [11,12]. To avoid the poly-Si gate depletion effects, the metal gate technology is used in this work. For better comparison among the devices, the threshold voltage $\left(V_{t h}\right)$ is kept at an approximate value for all device cases by carefully tuning the gate metal work function.

\section{Performance evaluation}

Fig. 2(a) shows the variation of electrostatic potential in the spacer and channel region (on state, $V_{D S}=0.7 \mathrm{~V}$ ) for the conventional and the proposed hybrid FinFETs with increasing high- $k$ spacer length $\left(L_{h k}\right)$. It is noticed that the potential lines diverge from their path and give extra peaks at the two spacer interface near the drain side in case of hybrid FinFET.

As the inner high- $k$ spacer length increases, the potential lines are well confined with an increase amount in the channel region and interface of the two spacers, which conclusively enhances the on-current $\left(I_{o n}\right)$ of the device. Similarly from Fig. 2(b), a significant improvement in SS (roughly 3.14\%) can be observed in case of hybrid FinFET. D2 device gives the optimum SS value i.e., $63.52 \mathrm{mV} /$ decade, which is nearer to the ideal value (60 $\mathrm{mV} /$ decade) and the degradation occurs with decrease in $L_{h k}$.

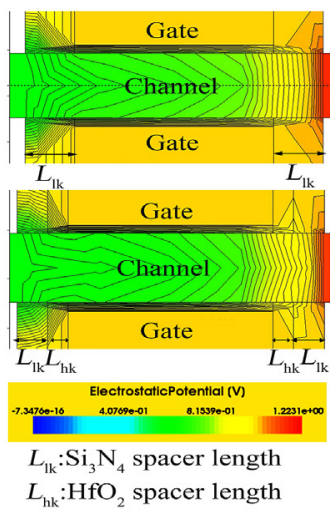

(a)

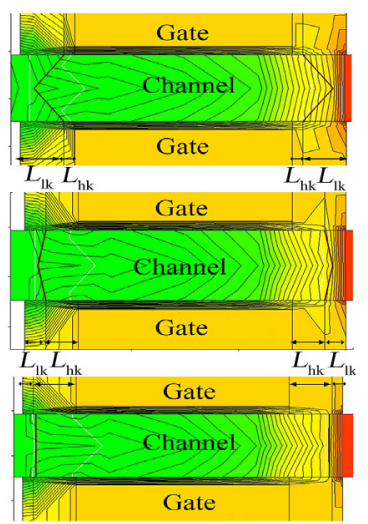

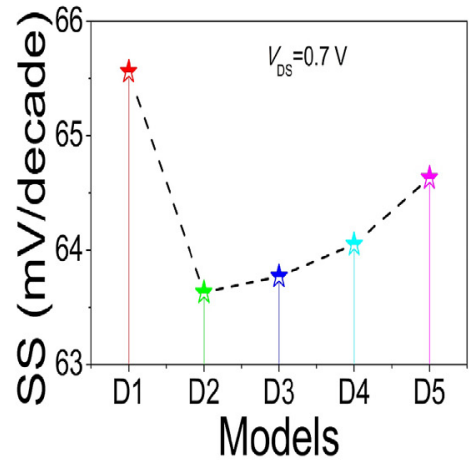

(b)

Fig. 2. Variation of electrostatic potential inside the channel and interface of the two spacers for conventional and hybrid FinFET for different $L_{l k}: L_{h k}$ ratios at $V_{D S}=0.7 \mathrm{~V}$. 

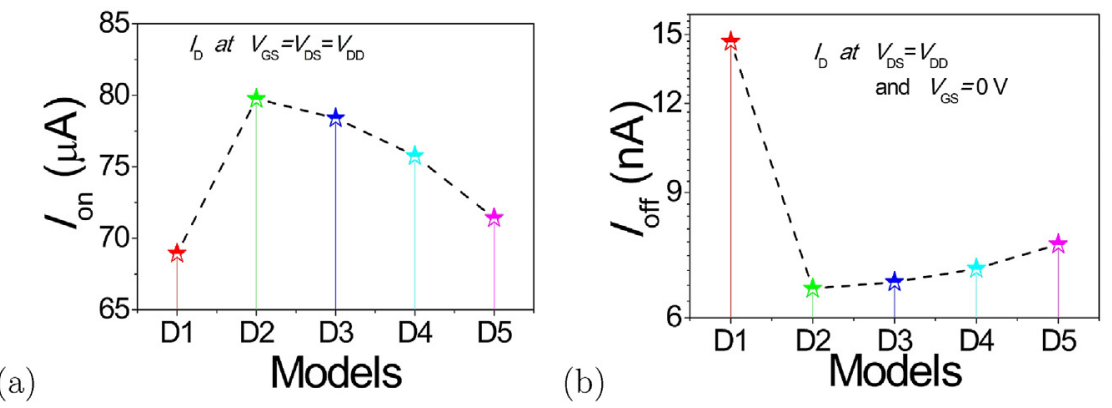

Fig. 3. Comparison among various architectures (a) $I_{\text {on }}$ (b) $I_{\text {off }}$.
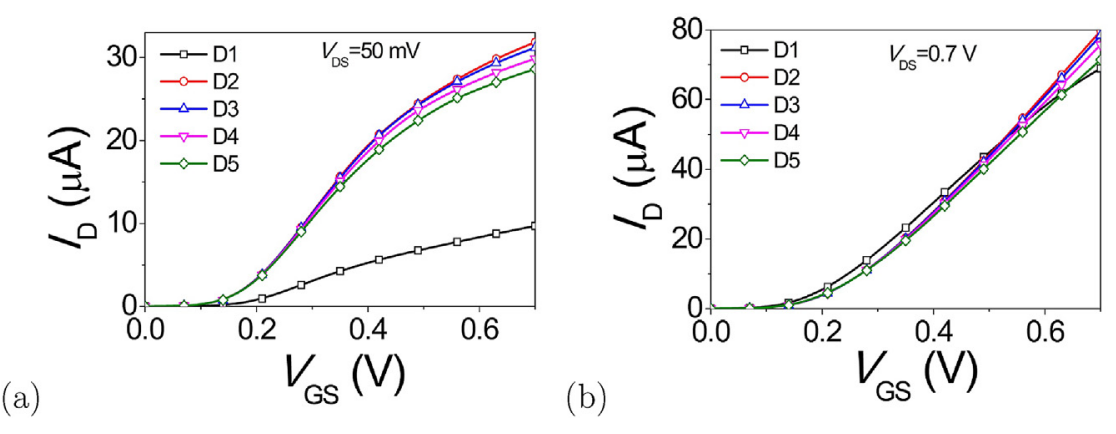

Fig. 4. Non-normalized $I_{D}-V_{G S}$ performance analysis between conventional SOI FinFET and hybrid FinFETs at different $L_{l k}: L_{h k}$ ratios for (a) $V_{D S}=50$ mV (b) $V_{D S}=0.7 \mathrm{~V}$.

An identical analogy can be made for $I_{\text {on }}$ and $I_{\text {off }}$ of our proposed device, which are presented in Fig. 3(a) and (b). The nonnormalized on-current is compared between conventional FinFET and different topologies of hybrid channel FinFET in Fig. 3(a). The drive current of any device plays an important role in HP consumer applications. Hence, it is crucial to perceive the response of inner high-k spacer length $\left(L_{h k}\right)$ on $I_{o f f}$ and $I_{o n}$. A sharp increase in $I_{o n}$ is identified with increase in $L_{h k}$ up to an optimum value of $4 \mathrm{~nm}$. The D2 ( $\left.L_{l k}: L_{h k}=1: 4\right)$ architecture exhibits highest enhancement in $I_{o n}$ (around 1.23x) from the conventional one i.e., D1. This increase in $I_{o n}$ is partially because of the UTB (hybrid channel) and due to the inclusion of larger $L_{h k}$, which further modulates the carrier concentration in underlap region. Comparative analysis of $I_{o f f}$ for different architectures is given in Fig. 3(b). The hybrid FinFETs with $L_{l k}: L_{h k}=1: 4$ shows an almost $60 \%$ of reduction in off-state leakage current as compared to conventional one.

Fig. 4(a) and (b) compare the transfer characteristic among conventional and hybrid FinFETs at different $L_{l k}: L_{h k}$ ratios for both low and high $V_{D S}$. It can be observed that the inclusion of dual-k spacer over the underlap regions of the proposed hybrid FinFETs can able to deliver 66.66\% higher non-normalized drive current with the same chip area as compared to conventional FinFET. Each nanometer increase in $L_{h k}$ spacer of the hybrid FinFET leads to a increase in $I_{o n}$. This enhancement in $I_{o n}$ with $L_{h k}$ is because of the gate fringe induced barrier lowering (GFIBL) in the underlap region [18].

(a)

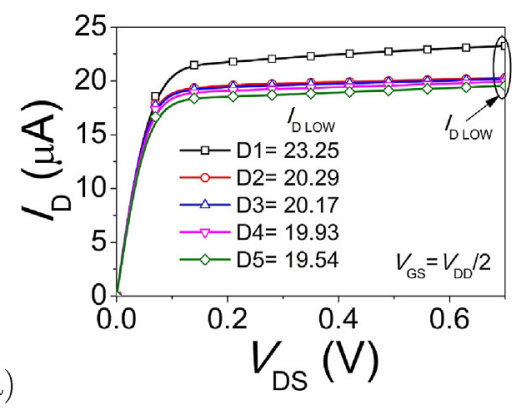

(b)

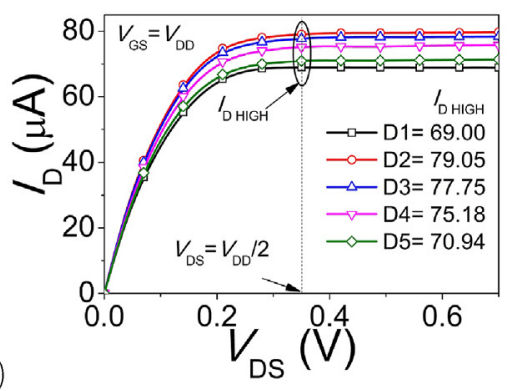

Fig. 5. Non-normalized $I_{D}-V_{D S}$ performance comparison among conventional SOI FinFET and hybrid FinFETs at different $L_{l k}: L_{h k}$ ratios for (a) $V_{G S}=V_{D D} / 2$ (b) $V_{D S}=V_{D D}$ 

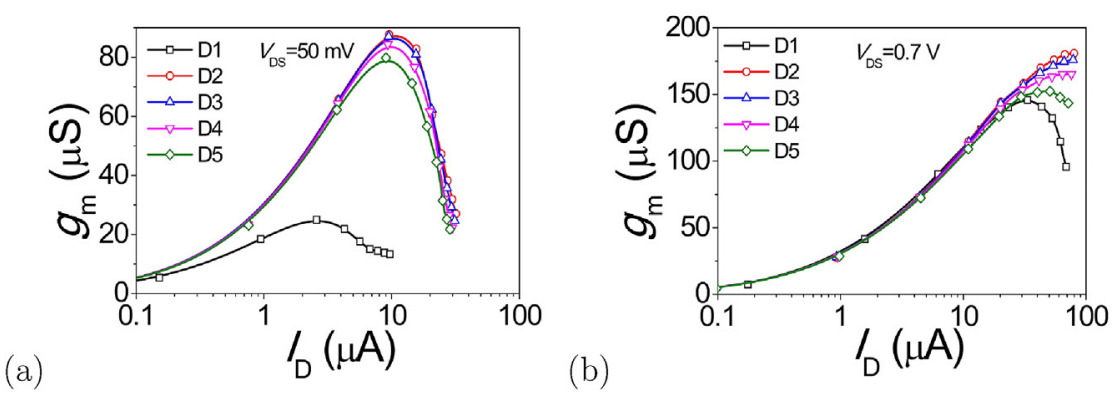

Fig. 6. $g_{m}-I_{D}$ performance analysis between conventional SOI FinFET and hybrid FinFETs at different $L_{l k}: L_{h k}$ ratios for (a) $V_{D S}=50 \mathrm{mV}$ (b) $V_{D S}=0.7 \mathrm{~V}$.

The $I_{D}-V_{D S}$ of the hybrid FinFETs with spacer technology are compared to the conventional FinFET at $V_{G S}=V_{D D} / 2$ and $V_{G S}=V_{D D}$ as shown in Fig. 5(a) and (b) respectively. $I_{D L O W}\left(V_{G S}=V_{D D} / 2, V_{D S}=V_{D D}\right)$ and $I_{D H I G H}\left(V_{G S}=V_{D D}, V_{D S}=V_{D D} / 2\right)$ are calculated to evaluate the effective drain current $\left(I_{D E F F}=\left(I_{D L O W}+I_{D H I G H}\right) / 2\right)$, which estimates the effective drain current drawn during switching. It is more realistic and way less optimistic than $I_{D S a t}$ assessment. By observing the inset values of $I_{D L O W}$ and $I_{D H I G H}$ from Fig. 5 , it is clear that the device having high $L_{h k}$ provide more $I_{D E F F}$. The D2 device $\left(L_{l k}: L_{h k}=1: 4\right)$ delivers $I_{D E F F}=(20.29+79.05) / 2 \mu \mathrm{A}=49.67 \mu \mathrm{A}$, which is optimum from any other cases and is nearly $7.6 \%$ higher than the conventional FinFET (D1).

Fig. 6(a) and (b) show the plot of $g_{m}$ for a conventional FinFET and different topologies of hybrid FinFETs with varying $L_{l k}: L_{h k}$ ratio at high $\left(V_{D S}=0.7 \mathrm{~V}\right)$ and low $\left(V_{D S}=50 \mathrm{mV}\right)$. To analyze the immense improvement in $g_{m}\left(\partial I_{D} / \partial V_{G S}\right)$ with an increase in $L_{l k}: L_{h k}$ spacer ratio, we have evaluated and studied the $I_{D}-g_{m}$ curve. According to the literature, access resistance problem is more serious in conventional trigate FinFETs. However, some solutions are available like increasing the $H_{F i n}$ out of the gate region [25]. The parasitic resistance problem can be avoided by using a UTB in FinFET technology, i.e., hybrid FinFETs with higher $L_{h k}$ spacer length, which further increases the drain current. This is also validated from Figs. 4-6, both the parameters i.e., on-current, $I_{D E F F}$, and $g_{m}$ are increasing with the increase in $L_{h k}$ in hybrid FinFETs. This is primarily due to the fringing field induced inversion charge modulation inside the underlap region. The charge-based model of $g_{m}$ for undoped multigate MOSFETs are represented as [26]:

$$
g_{m}=\partial I_{D} / \partial V_{G S}=\left(\mu W / L_{e f f}\right)\left(Q_{S}-Q_{D}\right)
$$

where $Q_{D}$ and $Q_{S}$ represent the gate-to-drain and gate-to-source charges, $\mu$ is the electron mobility, $W$, and $L_{e f f}$ are the width and effective channel length of the device. From Eq. (1), the $g_{m}$ is directly related to the source/drain charge difference $\left(Q_{S}-Q_{D}\right)$ and $\mu$. In subthreshold region (low $V_{D S}$ and $V_{G S}$ ), the $Q_{S}$ of the hybrid FinFETs increases moderately because of the immense gate control and in the mean time $Q_{D}$ remains almost constant as the gate induced fringing field is negligible. Hence, $Q_{S}-Q_{D}$ increases rapidly resulting a high subthreshold $g_{m}$ (Fig. 6(a)). Similarly, with an increase in $L_{h k}$, the reverse gate-todrain field plays a significant role, which further reduces the $Q_{D}$. As a result, $g_{m}$ increases with increase in $L_{h k}$ in subthreshold region and shows maximum for D2 device case $\left(L_{l k}: L_{h k}=1: 4\right)$. But, in superthreshold region (high $\left.V_{D S}\right)$, the $\mu$ term is very high and dominates $g_{m}$. Again, increasing $L_{h k}$ in superthreshold region, the $Q_{D}$ escalates with a unchanged $Q_{S}$ that reduces $Q_{S}-Q_{D}$ term of Eq. (1). However, due to the improved mobility for high $L_{h k}$ devices, there is a minor increment in $g_{m}$ can be noticed at superthreshold region (Fig. 6(b)).

Fig. 7 (a) illustrates the output conductance $\left(g_{d}\right)$ for conventional and hybrid FinFETs at $V_{G S}=0.7 \mathrm{~V}$. The $g_{d}$ can be expressed as [26].


Fig. 7. Analog parameters analyzed among hybrid FinFETs with different $L_{l k}: L_{h k}$ ratios and conventional FinFET (a) Output conductance ( $\left.g_{d}\right)$ at $V_{G S}=0.7 \mathrm{~V}(\mathrm{~b})$ gain $\left(g_{m} / g_{d}\right)$. 

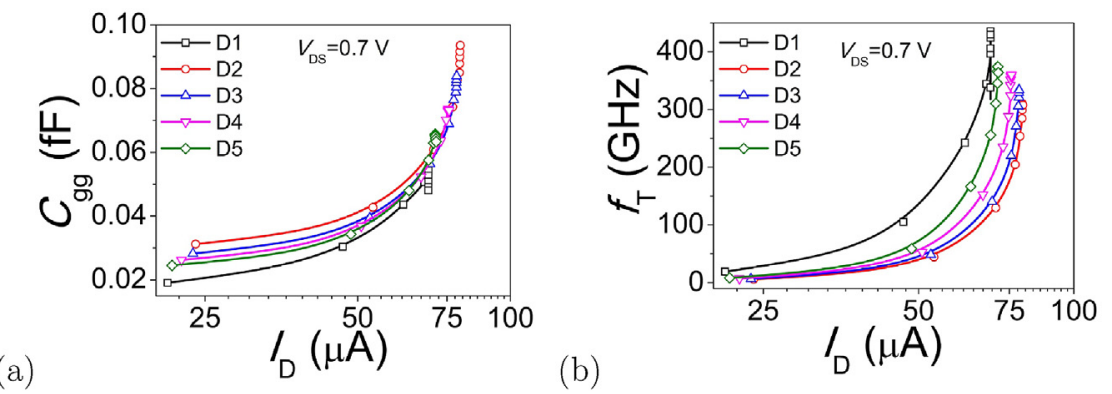

Fig. 8. Comparison of (a) $C_{g g}-I_{D}$ (b) $f_{T}-I_{D}$, among hybrid FinFETs with different $L_{l k}: L_{h k}$ ratios and conventional FinFET at $V_{D S}=0.7 \mathrm{~V}$.

$$
g_{d}=\partial I_{D} / \partial V_{D S}=\left(\mu W / L_{e f f}\right) Q_{D}
$$

The interpretation of above variables are well explained under Eq. (1). From Eq. (2), $g_{d}$ increases proportionately with $Q_{D}$. In earlier analogy under Fig. $6(\mathrm{~b})$, we have perceived that $Q_{D}$ plays a significant role in superthreshold region (high $V_{D S}$ ) and become the dominating factor for high $L_{h k}$ devices. So, it is observed from Fig. 7(a) that with increase in $L_{h k}$, the gate induced fringing field increases, which further enhances the $Q_{D}$ and increases the $g_{d}$ in superthreshold region. However, there is a reverse effect in subthreshold region i.e., $g_{d}$ decreases with increase in $L_{h k}$ as $Q_{D}$ remains constant at subthreshold region. For analog performances, the gain $\left(A_{V}=g_{m} / g_{d}\right)$ of the device is an important figure of merits (FOMs) that is analyzed in Fig. 7(b). From Fig. 7(b), in subthreshold region, $A_{V}$ is dominated by $g_{m}$ as a result, $A_{V}$ increases with increase in $L_{h k}$ that is due to higher $g_{m}$ as discussed in Fig. 6. And just a reverse phenomena is noticed in the superthreshold region. This is because of the $g_{d}$ factor as argued in Fig. 7(a).

The variation of total gate capacitance $\left(C_{g g}\right)$, and cutoff frequency $\left(f_{T}=g_{m} /\left(2 \pi C_{g g}\right)\right)$ with respect to $I_{D}$ for conventional FinFET and different architectures of hybrid FinFETs are shown in Fig. 8(a) and (b) respectively. It is seen that $C_{g g}$ increases with the increase in $I_{D}$, and the increment is higher for larger $L_{h k}$ hybrid FinFETs because of the advancement in the gate side wall developed fringing field lines. So, $C_{g g}$ increases whereas $g_{m}$ is almost constant for hybrid devices in the superthreshold region as shown in Fig. 6(b). Hence, the increase in $C_{g g}$ dominates the $f_{T}$ for the proposed devices consequently a decline in $f_{T}$ is observed in case of hybrid devices as demonstrated in Fig. 8(b).

\section{Conclusion}

This paper illustrates the benefits and drawbacks of hybrid FinFETs (UTB + FinFET + Spacer engineering) with compare to conventional FinFETs for subsequent technology nodes. The impact of dual- $k$ spacers (inner high- $k\left(\mathrm{HfO}_{2}\right)$ and outer low- $k$ $\left(\mathrm{Si}_{3} \mathrm{~N}_{4}\right)$ ) in improving the performances of the hybrid devices have been analyzed. The proposed device with an optimal high$k$ spacer length $\left(L_{h k}\right)$ can be efficiently reduce the SS and $I_{\text {off }}$ with an improved $I_{o n}$. The D2 topology i.e., hybrid FinFET with $L_{l k}: L_{h k}=1: 4$ exhibits superior electrostatic integrity in both $I_{o n}$ and $I_{o f f}$, which in turn enhance the overall device performance. Nonetheless, D2 architecture shows a $\mathbf{1 . 2 3 x}$ improvement in $I_{\text {on }}$, and around $60 \%$ reduction in $I_{\text {off }}$ as compared to SOI FinFET that is required for high speed, and low power consumption applications. We have also demonstrated the influence of $L_{l k}: L_{h k}$ ratios on various analog/RF FOMs like $g_{m}, A_{V}$, and $f_{T}$ in both subthreshold and superthreshold region of operations. From the presented outcomes, it is concluded that the hybrid FinFETs with higher $L_{l k}: L_{h k}$ ratios are outperformed as compared to conventional FinFET with regard to $I_{\text {off, }} I_{o n}$ and $g_{m}$. However, the primary drawbacks of these structures are the higher $C_{g g}$ that further pay the cost as a poor $f_{T}$.

\section{References}

[1] S.K. Saha, Compact Models for Integrated Circuit Design: Conventional Transistors and Beyond, CRC Press, 2015.

[2] Globalfoundries Looks Leapfrog Fab Rivals with New Process, Tech. rep, 2012.

[3] J. Kedzierski, M. Ieong, T. Kanarsky, Y. Zhang, H. Wong, Fabrication of metal gated finfets through complete gate silicidation with ni, Electron Devices IEEE Trans. 51 (12) (2004) 2115-2120.

[4] Y. Li, H.-M. Chou, J.-W. Lee, Investigation of electrical characteristics on surrounding-gate and omega-shaped-gate nanowire finfets, Nanotechnol. IEEE Trans. 4 (5) (2005) 510-516.

[5] D. Hisamoto, W.-C. Lee, J. Kedzierski, H. Takeuchi, K. Asano, C. Kuo, E. Anderson, T.-J. King, J. Bokor, C. Hu, Finfet-a self-aligned double-gate mosfet scalable to $20 \mathrm{~nm}$, Electron Devices IEEE Trans. 47 (12) (2000) 2320-2325.

[6] S.K. Mohapatra, K.P. Pradhan, D. Singh, P.K. Sahu, The role of geometry parameters and fin aspect ratio of sub-20nm SOI-FinFET: an analysis towards analog and RF circuit design, Nanotechnol. IEEE Trans. 14 (3) (2015) 546-554.

[7] P.K. Pal, B.K. Kaushik, S. Dasgupta, Investigation of symmetric dual-(k) spacer trigate FinFETs from delay perspective, Electron Devices IEEE Trans. 61 (11) (2014) 3579-3585.

[8] The International Technology Roadmap for Semiconductors, Tech. rep, 2013. http://public.itrs.net.

[9] H.M. Fahad, C. Hu, M.M. Hussain, Simulation study of a 3-D device integrating FinFET and UTBFET, Electron Devices IEEE Trans. 62 (1) (2015) $83-87$. 
[10] M.L. Lee, E.A. Fitzgerald, M.T. Bulsara, M.T. Currie, A. Lochtefeld, Strained Si, SiGe, and Ge channels for high-mobility metal-oxide-semiconductor fieldeffect transistors, J. Appl. Phys. 97 (1) (2005), 011101-1-011101-28.

[11] K.P. Pradhan, Priyanka, Mallikarjunarao, P.K. Sahu, Exploration of symmetric high-k spacer (SHS) hybrid FinFET for high performance application, Superlattices Microstruct. 90 (2016) 191-197.

[12] S.H. Park, Y. Liu, N. Kharche, M.S. Jelodar, G. Klimeck, M.S. Lundstrom, M. Luisier, Performance comparisons of III-V and strained-Si in planar FETs and nonplanar FinFETs at ultrashort gate length (12 nm), Electron Devices IEEE Trans. 59 (8) (2012) $2107-2114$.

[13] K. Pradhan, Priyanka, P. Sahu, Investigation of asymmetric high-k underlap spacer (ahus) hybrid finfet from temperature perspective, Microsyst. Technol. $1-6$.

[14] W. Zhang, J.G. Fossum, L. Mathew, The ITFET: a novel FinFET-based hybrid device, Electron Devices IEEE Trans. 53 (9) (2006) 2335 -2343.

[15] H.M. Fahad, A.M. Hussain, G.T. Sevilla, M. Hussain, Wavy channel transistor for area efficient high performance operation, Appl. Phys. Lett. 102 (13) (2013) 134109.

[16] K. Pradhan, P. Sahu, Benefits of asymmetric underlap dual-k spacer hybrid finfet over bulk finfet, IET Circuits Devices Syst 10 (5) (2016) $441-447$.

[17] K. Pradhan, Priyanka, P. Sahu, Temperature dependency of double material gate oxide (dmgo) symmetric dual-k spacer (sds) wavy finfet, Superlattices Microstruct. 89 (2016) 355-361.

[18] A.B. Sachid, C. Manoj, D.K. Sharma, V. Ramgopal Rao, Gate fringe-induced barrier lowering in underlap FinFET structures and its optimization, Electron Device Lett. IEEE 29 (1) (2008) 128-130.

[19] V. Trivedi, J.G. Fossum, M.M. Chowdhury, Nanoscale FinFETs with gate-source/drain underlap, Electron Devices IEEE Trans. 52 (1) (2005) 56-62.

[20] K. Koley, A. Dutta, B. Syamal, S.K. Saha, C.K. Sarkar, Subthreshold analog/rf performance enhancement of underlap dg fets with high-k spacer for low power applications, Electron Devices IEEE Trans. 60 (1) (2013) 63-69.

[21] P.K. Pal, B.K. Kaushik, S. Dasgupta, High-performance and robust SRAM cell based on asymmetric dual-k spacer FinFETs, Electron Devices IEEE Trans, 60 (10) (2013) 3371-3377.

[22] C. Hu, SOI and nanoscale MOSFETs, in: Device Research Conference, 2001, IEEE, 2001, pp. 3-4.

[23] C. Fiegna, Y. Yang, E. Sangiorgi, A.G. O'Neill, Analysis of self-heating effects in ultrathin-body SOI MOSFETs by device simulation, Electron Devices IEEE Trans. 55 (1) (2008) 233-244.

[24] T. Sentaurus, G. Version, Synopsys Inc, Mountain View, CA.

[25] A. Kranti, G.A. Armstrong, Design and optimization of finfets for ultra-low-voltage analog applications, Electron Devices IEEE Trans. 54 (12) (2007) $3308-3316$

[26] K. Koley, A. Dutta, S.K. Saha, C.K. Sarkar, Analysis of high- spacer asymmetric underlap dg-mosfet for soc application, Electron Devices IEEE Trans. 62 (6) (2015) 1733-1738. 